\title{
Comparative Assessment of the Water Quality of Four Types of Aquaculture Ponds under Different Culture Systems
}

\author{
Moshood Keke Mustapha
}

Department of Zoology, University of Ilorin, Ilorin, Nigeria

\author{
Accepted December, 2017
}

\begin{abstract}
Water quality of collapsible, concrete, earthen and natural ponds under different culture systems were assessed using of Photometer and tester. Temporal and spatial replications of samples were done in triplicates. Dissolved oxygen ranged between 4.6 to $6.8 \mathrm{mg} / \mathrm{l}$, carbon dioxide 1.4 - $3.0 \mathrm{mg} / \mathrm{l}$, nitrate 1.6 - $3.2 \mathrm{mg} / \mathrm{l}$, phosphate 0.8 - $2.3 \mathrm{mg} / \mathrm{l}$, calcium hardness 65- $100 \mathrm{mg} / \mathrm{l}$, magnesium hardness $30-50 \mathrm{mg} / \mathrm{l}$ and total hardness 80 and $165 \mathrm{mg} / \mathrm{l}$. Conductivity ranged between 346 - $472 \mu \mathrm{S} / \mathrm{cm}$, total dissolved solids 232 - $316 \mathrm{mg} / \mathrm{l}$, transparency 36 - $82 \mathrm{~cm}$, alkalinity 105 - $245 \mathrm{mg} / \mathrm{l}, \mathrm{pH}$ $6.35-8.03$ and temperature 29.1 to $35.9{ }^{\circ} \mathrm{C}$. Significant difference $(P<0.05)$ was obtained among the parameters in the ponds showing the effects of the different culture systems on the water quality. Variations in the water quality was due to the presence of plankton and macrophytes found in earthen and natural ponds where semi-intensive and extensive culture were practiced, use of artificial feed in collapsible and concrete ponds where intensive culture was done, effects of respiration, photosynthesis and decomposition, source of water and materials used for the construction of the ponds. Water quality in the ponds under the different culture systems was good.
\end{abstract}

Key words: fish, intensive, semi-intensive, extensive, parameters.

\section{Introduction}

The future of man's animal protein needs and demands will come from aquaculture especially fish farming. Fish are usually cultured in different water holding facilities such as ponds, tanks, cages, raceways, pens, happas etc. Successful fish culture depends to a large extent on the water quality of the holding facilities. Poor water quality in fish ponds will lead to poor fish health, welfare, growth, fillet quality, low profit and ultimately low production.

Water quality describes physical, chemical, biological and aesthetic properties of water which determine its fitness for use and its ability to maintain the health of farmed aquatic organisms. It is therefore imperative to have all the physical, chemical and biological factors in the ponds in a

\section{* Corresponding author: Moshood Mustapha, moonstapha@yahoo.com}

(c) BY-NC-ND $\odot 2017$ Moshood Mustapha, published by De Gruyter Open This work was licensed under the Creative Commons Attribution-NonCommercialNoDerivs 3.0 License balanced proportion and tolerable limit for optimum fish production. Poor water quality can be found in any water source used for fish culture, any type of pond or any type of culture system used in aquaculture.

The interactions of physico-chemical properties of water such as temperature, transparency, $\mathrm{pH}$, dissolved oxygen, alkalinity, hardness, organic and inorganic ions as well as biological factors like plankton, aquatic microphytes control the success of fish production in any culture system be it intensive, semi-intensive or extensive. In the same vein, the culture systems could influence the physico-chemistry and biological properties of the water and hence the water quality. Water quality in fish ponds and aquaculture has been comprehensively reviewed by Alabaster and Lloyd (1982), Boyd (1998), Boyd \& Tucker (1998), Zweig et al. (1999), Bhatnagar \& Devi (2013), among several other workers $[1,2,3,4,5]$.

The objective of this paper is to assess some physico-chemical factors which constitute water 
quality in four different types of aquaculture ponds under different culture systems.

\section{Material and Methods}

Water quality of four different aquaculture ponds under different culture systems were assessed weekly for 12 weeks between February and April 2015. The ponds were of the same size and dimension of $4.5 \mathrm{~m} \times 4.5 \mathrm{~m} \times 1 \mathrm{~m}(20 \mathrm{~m} 2 \times 1 \mathrm{~m})$, volume capacity of $20 \mathrm{~m} 3$ (20000 litres) and containing a population of 2000 Clarias gariepinus with biomass of $500 \pm 50 \mathrm{~g}$. The ponds were collapsible pond, concrete pond, earthen pond and a natural pond. Borehole or ground water was the source of water to the collapsible pond, concrete pond and earthen pond while the natural pond has its water source from the rains and runoff from the watershed. Intensive system of fish culture was carried out in both collapsible and concrete ponds; semi-intensive system was practiced in earthen pond, while extensive system was done in natural pond.

The ponds are located in Ilorin, Nigeria, on latitude $8^{\circ} 30^{\prime}$ and $8^{\circ} 50^{\prime} \mathrm{N}$ and longitude $4^{\circ} 20^{\prime}$ and $4^{\circ} 35^{\prime} \mathrm{E}$. The city is situated in the transitional zone within the forest and the guinea savannah regions of Nigeria. The climate is tropical under the influence of the two trade winds prevailing in Nigeria with two climatic seasons i.e. rainy and dry season. The rainy season is between March and November and the annual rainfall varies from $1000 \mathrm{~mm}$ to $1500 \mathrm{~mm}$, with the peak between September and early October. The mean monthly temperature is generally high $\left(27.5^{\circ} \mathrm{C}\right)$.

The soil type is Luvisols, Acrisols, Ferralsols and Lithosols and the soils are physically fragile containing a large proportion of sand having being derived from interne and lacustrine deposits.

The monthly average daily total of diffuse solar radiation is highest in February due to Harmattan haze and thin clouds, and lowest in November. On an annual average, the diffuse component constituted about $60 \%$ of the global solar radiation.

$50 \mathrm{ml}$ plastic water bottle each was used for the collection of water samples in the afternoons at $1.00 \mathrm{pm}$ for the analysis of the following water quality parameters. Alkalinity, calcium and magnesium hardness were measured using colorimetric method, phosphate was measured using amino acid method, nitrate by cadmium reduction method, carbon dioxide by phenolphthalein titrimetric method and dissolved oxygen by azide modification of winkler's method. All the parameters were analysed with the aid of Hanna Multiparameter Bench Photometer for Laboratories Model HI 83200-02. Temperature, $\mathrm{pH}$, electrical conductivity and total dissolved solids were measured in situ in the ponds using Hanna Portable pH/EC/TDS/Temp combined waterproof tester Model HI 98129. Transparency was evaluated by extinction method with the use of secchi disc. Temporal and spatial replications of samples were done with triplicates water samples of the ponds obtained and measured for all the chemical parameters, while $\mathrm{pH}$ and temperature, electrical conductivity and total dissolved solids measurements were also done temporally and spatially and replicated with the parameters measured in triplicates in situ.

Statistical Analyses

GLM procedure of statistical analysis system 9.1.3 [6] was used to analyse the results. Weekly mean difference of each triplicate parameter was compared using two way ANOVA at $\mathrm{P}<0.05$ to see the variations due to weeks and ponds.

\section{Results and Discussions}

The result of the mean weekly variations in the water quality of the four types of aquaculture ponds is presented in Tables 1, 2, 3 and 4.

Concentration of dissolved oxygen in the four ponds ranged from the highest of $6.8 \mathrm{mg} / \mathrm{l}$ recorded in the natural pond to the lowest of 4.6 $\mathrm{mg} / \mathrm{l}$ obtained in collapsible pond. Carbon dioxide ranged from a highest concentration of $3.0 \mathrm{mg} / \mathrm{l}$ obtained in collapsible pond to the lowest of 1.4 $\mathrm{mg} / \mathrm{l}$ recorded in natural pond. Nitrate ranged between $3.2 \mathrm{mg} / \mathrm{l}$ and $1.6 \mathrm{mg} / \mathrm{l}$ with the highest concentration obtained in collapsible pond in week 12 and the lowest in natural pond in week 1 . The range of phosphate concentrations in the ponds was between highest of $2.3 \mathrm{mg} / \mathrm{l}$ recorded in collapsible pond in week 12 and $0.8 \mathrm{mg} / \mathrm{l}$ obtained from natural pond in week 1 . Highest concentrations of calcium and magnesium hardness was $100 \mathrm{mg} / \mathrm{l}$ and $65 \mathrm{mg} / \mathrm{l}$ respectively recorded from collapsible pond in week 12 , while lowest concentrations of $50 \mathrm{mg} / \mathrm{l}$ and $30 \mathrm{mg} / \mathrm{l}$ respectively was obtained in natural pond in week 1. Collapsible pond recorded the highest concentration of electrical conductivity with a value of $472 \mu \mathrm{S} / \mathrm{cm}$ in week 12 , while the lowest value of $346 \mu \mathrm{S} / \mathrm{cm}$ was obtained from natural pond in week 1 . The same trend was obtained for total dissolved solids with the highest concentration of $316 \mathrm{mg} / \mathrm{l}$ obtained in week 12 from collapsible pond and lowest concentration of $232 \mathrm{mg} / \mathrm{l}$ was recorded in natural pond in week 1 . Transparency fluctuated between the highest of $82 \mathrm{~cm}$ in collapsible pond obtained in week 1 and lowest of $36 \mathrm{~cm}$ recorded from natural pond in week 12 . Alkalinity ranged from 105 to $245 \mathrm{mg} / \mathrm{l}$ with the highest obtained in collapsible pond and lowest recorded in natural pond. 
Mean weekly variations in the water quality of collapsible pond

Table 1

\begin{tabular}{|c|c|c|c|c|c|c|c|c|c|c|c|c|c|}
\hline Week & Temp & $\begin{array}{l}\text { DO } \\
\mathrm{mg} / \mathrm{l}\end{array}$ & $\begin{array}{l}\mathrm{CO}_{2} \\
\mathrm{mg} / \mathrm{l}\end{array}$ & $\begin{array}{l}\mathrm{N} \\
\mathrm{mg} / \mathrm{l}\end{array}$ & $\begin{array}{l}\mathrm{P} \\
\mathrm{mg} / \mathrm{l}\end{array}$ & $\begin{array}{l}\mathrm{Ca} \\
\mathrm{mg} / \mathrm{l}\end{array}$ & $\begin{array}{l}\mathrm{Mg} \\
\mathrm{mg} / \mathrm{l}\end{array}$ & $\begin{array}{l}\mathrm{TH} \\
\mathrm{mg} / \mathrm{l}\end{array}$ & $\begin{array}{l}E C \\
\mu S / c m\end{array}$ & $\begin{array}{l}\text { TDS } \\
\mathrm{mg} / \mathrm{l}\end{array}$ & $\begin{array}{l}\text { Transp } \\
\text { cm }\end{array}$ & $\mathrm{pH}$ & $\begin{array}{l}\text { Alk } \\
\text { mg/l }\end{array}$ \\
\hline 1 & $\begin{array}{c}31.9 \\
\pm 0.1\end{array}$ & $\begin{array}{l}6.0 \\
\pm 0.1\end{array}$ & $\begin{array}{l}3.0 \\
\pm 0.0\end{array}$ & $\begin{array}{l}2.2 \\
\pm 0.0\end{array}$ & $\begin{array}{l}1.6 \\
\pm 0.0\end{array}$ & $90 \pm 0.1$ & $\begin{array}{l}55 \\
\pm 0.1\end{array}$ & $\begin{array}{l}145 \\
\pm 0.2\end{array}$ & $\begin{array}{l}380 \\
\pm 0.1\end{array}$ & $255 \pm 0.1$ & $82 \pm 0.0$ & $\begin{array}{l}8.01 \\
\pm 0.2\end{array}$ & $\begin{array}{l}225 \\
\pm 0.2\end{array}$ \\
\hline 2 & $\begin{array}{l}32.5 \\
\pm 0.1\end{array}$ & $\begin{array}{l}5.8 \\
\pm 0.1\end{array}$ & $\begin{array}{l}3.0 \\
\pm 0.0\end{array}$ & $\begin{array}{l}2.3 \\
\pm 0.0\end{array}$ & $\begin{array}{l}1.6 \\
\pm 0.0\end{array}$ & $\begin{array}{l}990 \\
\pm 0.1\end{array}$ & $\begin{array}{l}55 \\
\pm 0.1\end{array}$ & $\begin{array}{l}145 \\
\pm 0.2\end{array}$ & $\begin{array}{l}386 \\
\pm 0.1\end{array}$ & $259 \pm 0.1$ & $82 \pm 0.0$ & $\begin{array}{l}8.01 \\
\pm 0.2\end{array}$ & $\begin{array}{l}225 \\
\pm 0.2\end{array}$ \\
\hline 3 & $\begin{array}{l}32.7 \\
\pm 0.1 \\
\end{array}$ & $\begin{array}{l}5.7 \\
\pm 0.1 \\
\end{array}$ & $\begin{array}{l}2.9 \\
\pm 0.0 \\
\end{array}$ & $\begin{array}{l}2.4 \\
\pm 0.0\end{array}$ & $\begin{array}{l}1.8 \\
\pm 0.0\end{array}$ & $100 \pm 0.1$ & $\begin{array}{l}55 \\
\pm 0.1\end{array}$ & $\begin{array}{l}155 \\
\pm 0.2\end{array}$ & $\begin{array}{l}392 \\
\pm 0.1 \\
\end{array}$ & $263 \pm 0.1$ & $\begin{array}{l}82 \\
\pm 0.0\end{array}$ & $\begin{array}{l}8.01 \\
\pm 0.2\end{array}$ & $\begin{array}{l}235 \\
\pm 0.2\end{array}$ \\
\hline 4 & $\begin{array}{l}32.8 \\
\pm 0.1\end{array}$ & $\begin{array}{l}5.6 \\
\pm 0.1\end{array}$ & $\begin{array}{l}2.8 \\
\pm 0.0\end{array}$ & $\begin{array}{l}2.5 \\
\pm 0.0\end{array}$ & $\begin{array}{l}1.8 \\
\pm 0.0\end{array}$ & $100 \pm 0.1$ & $\begin{array}{l}60 \\
\pm 0.1\end{array}$ & $\begin{array}{l}160 \\
\pm 0.2\end{array}$ & $\begin{array}{l}394 \\
\pm 0.1\end{array}$ & $264 \pm 0.1$ & $\begin{array}{l}80 \\
\pm 0.0\end{array}$ & $\begin{array}{l}8.02 \\
\pm 0.2\end{array}$ & $\begin{array}{l}240 \\
\pm 0.2\end{array}$ \\
\hline 5 & $\begin{array}{l}33.1 \\
\pm 0.1 \\
\end{array}$ & $\begin{array}{l}5.2 \\
\pm 0.1 \\
\end{array}$ & $\begin{array}{l}2.8 \\
\pm 0.0 \\
\end{array}$ & $\begin{array}{l}2.5 \\
\pm 0.0\end{array}$ & $\begin{array}{l}1.9 \\
\pm 0.0\end{array}$ & $100 \pm 0.1$ & $\begin{array}{l}60 \\
\pm 0.1 \\
\end{array}$ & $\begin{array}{l}160 \\
\pm 0.2 \\
\end{array}$ & $\begin{array}{l}395 \\
\pm 0.1 \\
\end{array}$ & $265 \pm 0.1$ & $\begin{array}{l}80 \\
\pm 0.0\end{array}$ & $\begin{array}{l}8.02 \\
\pm 0.2 \\
\end{array}$ & $\begin{array}{l}240 \\
\pm 0.2\end{array}$ \\
\hline 6 & $\begin{array}{l}33.4 \\
\pm 0.1 \\
\end{array}$ & $\begin{array}{l}5.1 \\
\pm 0.1 \\
\end{array}$ & $\begin{array}{l}2.6 \\
\pm 0.0 \\
\end{array}$ & $\begin{array}{l}2.6 \\
\pm 0.0 \\
\end{array}$ & $\begin{array}{l}2.0 \\
\pm 0.0 \\
\end{array}$ & $100 \pm 0.1$ & $\begin{array}{l}60 \\
\pm 0.1 \\
\end{array}$ & $\begin{array}{l}160 \\
\pm 0.2 \\
\end{array}$ & $\begin{array}{l}400 \\
\pm 0.1 \\
\end{array}$ & $268 \pm 0.1$ & $\begin{array}{l}80 \\
\pm 0.0 \\
\end{array}$ & $\begin{array}{l}8.02 \\
\pm 0.2 \\
\end{array}$ & $\begin{array}{l}240 \\
\pm 0.2 \\
\end{array}$ \\
\hline 7 & $\begin{array}{l}33.8 \\
\pm 0.1\end{array}$ & $\begin{array}{l}5.0 \\
\pm 0.1\end{array}$ & $\begin{array}{l}2.5 \\
\pm 0.0\end{array}$ & $\begin{array}{l}2.8 \\
\pm 0.0\end{array}$ & $\begin{array}{l}2.0 \\
\pm 0.0\end{array}$ & $100 \pm 0.1$ & $\begin{array}{l}60 \\
\pm 0.1\end{array}$ & $\begin{array}{l}160 \\
\pm 0.2\end{array}$ & $\begin{array}{l}408 \\
\pm 0.1\end{array}$ & $273 \pm 0.1$ & $\begin{array}{l}81 \\
\pm 0.0\end{array}$ & $\begin{array}{l}8.02 \\
\pm 0.2\end{array}$ & $\begin{array}{l}240 \\
\pm 0.2\end{array}$ \\
\hline 8 & $\begin{array}{l}34.3 \\
\pm 0.1\end{array}$ & $\begin{array}{l}5.0 \\
\pm 0.1\end{array}$ & $\begin{array}{l}2.5 \\
\pm 0.0\end{array}$ & $\begin{array}{l}2.8 \\
\pm 0.0\end{array}$ & $\begin{array}{l}2.1 \\
\pm 0.0\end{array}$ & $100 \pm 0.1$ & $\begin{array}{l}60 \\
\pm 0.1\end{array}$ & $\begin{array}{l}160 \\
\pm 0.2\end{array}$ & $\begin{array}{l}426 \\
\pm 0.1\end{array}$ & $285 \pm 0.1$ & $\begin{array}{l}81 \\
\pm 0.0\end{array}$ & $\begin{array}{l}8.02 \\
\pm 0.2\end{array}$ & $\begin{array}{l}240 \\
\pm 0.2\end{array}$ \\
\hline 9 & $\begin{array}{l}34.6 \\
\pm 0.1\end{array}$ & $\begin{array}{l}4.9 \\
\pm 0.1\end{array}$ & $\begin{array}{l}2.4 \\
\pm 0.0\end{array}$ & $\begin{array}{l}3.0 \\
\pm 0.0\end{array}$ & $\begin{array}{l}2.1 \\
\pm 0.0\end{array}$ & $100 \pm 0.1$ & $\begin{array}{l}65 \\
\pm 0.1\end{array}$ & $\begin{array}{l}165 \\
\pm 0.2\end{array}$ & $\begin{array}{l}44 \\
0 \pm 0.1\end{array}$ & $294 \pm 0.1$ & $\begin{array}{l}80 \\
\pm 0.0\end{array}$ & $\begin{array}{l}8.02 \\
\pm 0.2\end{array}$ & $\begin{array}{l}245 \\
\pm 0.2\end{array}$ \\
\hline 10 & $\begin{array}{l}35.0 \\
\pm 0.1\end{array}$ & $\begin{array}{l}4.8 \\
\pm 0.1\end{array}$ & $\begin{array}{l}2.3 \\
\pm 0.0\end{array}$ & $\begin{array}{l}3.0 \\
\pm 0.0\end{array}$ & $\begin{array}{l}2.2 \\
\pm 0.0\end{array}$ & $100 \pm 0.1$ & $\begin{array}{l}65 \\
\pm 0.1\end{array}$ & $\begin{array}{l}165 \\
\pm 0.2\end{array}$ & $\begin{array}{l}456 \\
\pm 0.1 \\
\end{array}$ & $306 \pm 0.1$ & $\begin{array}{l}80 \\
\pm 0.0\end{array}$ & $\begin{array}{l}8.03 \\
\pm 0.2\end{array}$ & $\begin{array}{l}245 \\
\pm 0.2\end{array}$ \\
\hline 11 & $\begin{array}{l}35.7 \\
\pm 0.1\end{array}$ & $\begin{array}{l}4.7 \\
\pm 0.1\end{array}$ & $\begin{array}{l}2.2 \\
\pm 0.0\end{array}$ & $\begin{array}{l}3.1 \\
\pm 0.0\end{array}$ & $\begin{array}{l}2.2 \\
\pm 0.0\end{array}$ & $100 \pm 0.1$ & $\begin{array}{l}65 \\
\pm 0.1\end{array}$ & $\begin{array}{l}165 \\
\pm 0.2\end{array}$ & $\begin{array}{l}468 \\
\pm 0.1\end{array}$ & $314 \pm 0.1$ & $\begin{array}{l}80 \\
\pm 0.0\end{array}$ & $\begin{array}{l}8.03 \\
\pm 0.2\end{array}$ & $\begin{array}{l}245 \\
\pm 0.2\end{array}$ \\
\hline 12 & $\begin{array}{l}35.9 \\
\pm 0.1\end{array}$ & $\begin{array}{l}4.6 \\
\pm 0.1\end{array}$ & $\begin{array}{l}2.2 \\
\pm 0.0\end{array}$ & $\begin{array}{l}3.2 \\
\pm 0.0\end{array}$ & $\begin{array}{l}2.3 \\
\pm 0.0\end{array}$ & $100 \pm 0.1$ & $\begin{array}{l}65 \\
\pm 0.1\end{array}$ & $\begin{array}{l}165 \\
\pm 0.2\end{array}$ & $\begin{array}{l}472 \\
\pm 0.1\end{array}$ & $316 \pm 0.1$ & $\begin{array}{l}80 \\
\pm 0.0\end{array}$ & $\begin{array}{l}8.03 \\
\pm 0.2\end{array}$ & $\begin{array}{l}245 \\
\pm 0.2\end{array}$ \\
\hline
\end{tabular}

Mean weekly variations in the water quality of concrete pond

Table 2

\begin{tabular}{|c|c|c|c|c|c|c|c|c|c|c|c|c|c|}
\hline Week & Temp & $\begin{array}{l}\text { DO } \\
\mathrm{mg} / \mathrm{l}\end{array}$ & $\begin{array}{l}\mathrm{CO}_{2} \\
\mathrm{mg} / \mathrm{l}\end{array}$ & $\begin{array}{l}\mathrm{N} \\
\mathrm{mg} / \mathrm{l}\end{array}$ & $\begin{array}{l}\mathrm{P} \\
\mathrm{mg} / \mathrm{l}\end{array}$ & $\begin{array}{l}\mathrm{Ca} \\
\mathrm{mg} / \mathrm{l}\end{array}$ & $\begin{array}{l}\mathrm{Mg} \\
\mathrm{mg} / \mathrm{l}\end{array}$ & $\begin{array}{l}\text { TH } \\
\mathrm{mg} / \mathrm{l}\end{array}$ & $\begin{array}{l}\text { EC } \\
\mu S / c m\end{array}$ & $\begin{array}{l}\text { TDS } \\
\mathrm{mg} / \mathrm{l}\end{array}$ & $\begin{array}{l}\text { Transp } \\
\text { cm }\end{array}$ & pH & $\begin{array}{l}\text { Alk } \\
\mathrm{mg} / \mathrm{l}\end{array}$ \\
\hline 1 & $\begin{array}{l}309 \\
\pm 01\end{array}$ & $\begin{array}{l}6.1 \\
\pm 0.1\end{array}$ & $\begin{array}{l}2.7 \\
\pm 0.0\end{array}$ & $\begin{array}{l}2.0 \\
\pm 0.0\end{array}$ & $\begin{array}{l}1.2 \\
\pm 0.0\end{array}$ & $90 \pm 0.1$ & $\begin{array}{l}45 \\
\pm 0.1\end{array}$ & $\begin{array}{l}135 \\
\pm 0.2\end{array}$ & $\begin{array}{l}358 \\
\pm 0.1\end{array}$ & $\begin{array}{l}240 \\
\pm 0.1\end{array}$ & $\begin{array}{l}77 \\
\pm 0.0\end{array}$ & $\begin{array}{l}7.88 \\
\pm 0.2\end{array}$ & $\begin{array}{l}200 \\
\pm 0.2\end{array}$ \\
\hline 2 & $\begin{array}{l}311 \\
\pm 01\end{array}$ & $\begin{array}{l}6.0 \\
\pm 0.1\end{array}$ & $\begin{array}{l}2.7 \\
\pm 0.0\end{array}$ & $\begin{array}{l}2.1 \\
\pm 0.0\end{array}$ & $\begin{array}{l}1.2 \\
\pm 0.0\end{array}$ & $90 \pm 0.1$ & $\begin{array}{l}45 \\
\pm 0.1\end{array}$ & $\begin{array}{l}135 \\
\pm 0.2\end{array}$ & $\begin{array}{l}364 \\
\pm 0.1\end{array}$ & $\begin{array}{l}244 \\
\pm 0.1\end{array}$ & $\begin{array}{l}78 \\
\pm 0.0\end{array}$ & $\begin{array}{l}7.88 \\
\pm 0.2\end{array}$ & $\begin{array}{l}205 \\
\pm 0.2\end{array}$ \\
\hline 3 & $\begin{array}{l}316 \\
\pm 01\end{array}$ & $\begin{array}{l}5.8 \\
\pm 0.1\end{array}$ & $\begin{array}{l}2.6 \\
\pm 0.0\end{array}$ & $\begin{array}{l}2.2 \\
\pm 0.0\end{array}$ & $\begin{array}{l}1.4 \\
\pm 0.0\end{array}$ & $90 \pm 0.1$ & $\begin{array}{l}50 \\
\pm 0.1\end{array}$ & $\begin{array}{l}14 \\
\pm 0.2\end{array}$ & $\begin{array}{l}370 \\
\pm 0.1\end{array}$ & $\begin{array}{l}248 \\
\pm 0.1\end{array}$ & $78 \pm 0.0$ & $\begin{array}{l}7.90 \\
\pm 0.2\end{array}$ & $\begin{array}{l}205 \\
\pm 0.2\end{array}$ \\
\hline 4 & $\begin{array}{l}319 \\
\pm 01\end{array}$ & $\begin{array}{l}5.7 \\
\pm 0.1\end{array}$ & $\begin{array}{l}2.4 \\
\pm 0.0\end{array}$ & $\begin{array}{l}2.3 \\
\pm 0.0\end{array}$ & $\begin{array}{l}1.5 \\
\pm 0.0\end{array}$ & $90 \pm 0.1$ & $\begin{array}{l}50 \\
\pm 0.1\end{array}$ & $\begin{array}{l}140 \\
\pm 0.2\end{array}$ & $\begin{array}{l}376 \\
\pm 0.1\end{array}$ & $\begin{array}{l}252 \\
\pm 0.1\end{array}$ & $76 \pm 0.0$ & $\begin{array}{l}7.90 \\
\pm 0.2\end{array}$ & $\begin{array}{l}205 \\
\pm 0.2\end{array}$ \\
\hline 5 & $\begin{array}{l}325 \\
\pm 01 \\
\end{array}$ & $\begin{array}{l}5.4 \\
\pm 0.1 \\
\end{array}$ & $\begin{array}{l}2.4 \\
\pm 0.0 \\
\end{array}$ & $\begin{array}{l}2.3 \\
\pm 0.0 \\
\end{array}$ & $\begin{array}{l}1.5 \\
\pm 0.0 \\
\end{array}$ & $90 \pm 0.1$ & $\begin{array}{l}50 \\
\pm 0.1\end{array}$ & $\begin{array}{l}140 \\
\pm 0.2 \\
\end{array}$ & $\begin{array}{l}384 \\
\pm 0.1 \\
\end{array}$ & $\begin{array}{l}257 \\
\pm 0.1 \\
\end{array}$ & $76 \pm 0.0$ & $\begin{array}{l}7.90 \\
\pm 0.2 \\
\end{array}$ & $\begin{array}{l}205 \\
\pm 0.2 \\
\end{array}$ \\
\hline 6 & $\begin{array}{l}331 \\
\pm 01 \\
\end{array}$ & $\begin{array}{l}5.2 \\
\pm 0.1 \\
\end{array}$ & $\begin{array}{l}2.2 \\
\pm 0.0\end{array}$ & $\begin{array}{l}2.4 \\
\pm 0.0 \\
\end{array}$ & $\begin{array}{l}1.6 \\
\pm 0.0 \\
\end{array}$ & $95 \pm 0.1$ & $\begin{array}{l}50 \\
\pm 0.1\end{array}$ & $\begin{array}{l}145 \\
\pm 0.2\end{array}$ & $\begin{array}{l}390 \\
\pm 0.1 \\
\end{array}$ & $\begin{array}{l}261 \\
\pm 0.1\end{array}$ & $75 \pm 0.0$ & $\begin{array}{l}8.00 \\
\pm 0.2 \\
\end{array}$ & $\begin{array}{l}205 \\
\pm 0.2\end{array}$ \\
\hline 7 & $\begin{array}{l}337 \\
\pm 01\end{array}$ & $\begin{array}{l}5.1 \\
\pm 0.1\end{array}$ & $\begin{array}{l}2.2 \\
\pm 0.0\end{array}$ & $\begin{array}{l}2.5 \\
\pm 0.0\end{array}$ & $\begin{array}{l}1.6 \\
\pm 0.0\end{array}$ & $95 \pm 0.1$ & $\begin{array}{l}50 \\
\pm 0.1\end{array}$ & $\begin{array}{l}145 \\
\pm 0.2\end{array}$ & $\begin{array}{l}398 \\
\pm 0.1\end{array}$ & $\begin{array}{l}267 \\
\pm 0.1\end{array}$ & $75 \pm 0.0$ & $\begin{array}{l}8.01 \\
\pm 0.2\end{array}$ & $\begin{array}{l}210 \\
\pm 0.2\end{array}$ \\
\hline 8 & $\begin{array}{l}340 \\
\pm 01\end{array}$ & $\begin{array}{l}5.0 \\
\pm 0.1\end{array}$ & $\begin{array}{l}2.1 \\
\pm 0.0\end{array}$ & $\begin{array}{l}2.6 \\
\pm 0.0\end{array}$ & $\begin{array}{l}1.8 \\
\pm 0.0\end{array}$ & $95 \pm 0.1$ & $\begin{array}{l}500 \\
\pm 0.1\end{array}$ & $\begin{array}{l}145 \\
\pm 0.2\end{array}$ & $\begin{array}{l}412 \\
\pm 0.1\end{array}$ & $\begin{array}{l}276 \\
\pm 0.1\end{array}$ & $\begin{array}{l}75 \\
\pm 0.0\end{array}$ & $\begin{array}{l}8.01 \\
\pm 0.2\end{array}$ & $\begin{array}{l}210 \\
\pm 0.2\end{array}$ \\
\hline 9 & $\begin{array}{l}341 \\
\pm 01\end{array}$ & $\begin{array}{l}5.0 \\
\pm 0.1\end{array}$ & $\begin{array}{l}2.0 \\
\pm 0.0\end{array}$ & $\begin{array}{l}2.8 \\
\pm 0.0\end{array}$ & $\begin{array}{l}1.8 \\
\pm 0.0\end{array}$ & $95 \pm 0.1$ & $\begin{array}{l}55 \\
\pm 0.1\end{array}$ & $\begin{array}{l}150 \\
\pm 0.2\end{array}$ & $\begin{array}{l}426 \\
\pm 0.1\end{array}$ & $\begin{array}{l}285 \\
\pm 0.1\end{array}$ & $76 \pm 0.0$ & $\begin{array}{l}8.01 \\
\pm 0.2\end{array}$ & $\begin{array}{l}210 \\
\pm 0.2\end{array}$ \\
\hline 10 & $\begin{array}{l}346 \\
\pm 01\end{array}$ & $\begin{array}{l}4.9 \\
\pm 0.1\end{array}$ & $\begin{array}{l}2.0 \\
\pm 0.0\end{array}$ & $\begin{array}{l}2.8 \\
\pm 0.0\end{array}$ & $\begin{array}{l}1.8 \\
\pm 0.0\end{array}$ & $95 \pm 0.1$ & $\begin{array}{l}55 \\
\pm 0.1\end{array}$ & $\begin{array}{l}150 \\
\pm 0.2\end{array}$ & $\begin{array}{l}430 \\
\pm 0.1\end{array}$ & $\begin{array}{l}288 \\
\pm 0.1\end{array}$ & $75 \pm 0.0$ & $\begin{array}{l}8.01 \\
\pm 0.2\end{array}$ & $\begin{array}{l}220 \\
\pm 0.2\end{array}$ \\
\hline 11 & $\begin{array}{l}348 \\
\pm 01\end{array}$ & $\begin{array}{l}4.9 \\
\pm 0.1\end{array}$ & $\begin{array}{l}2.0 \\
\pm 0.0\end{array}$ & $\begin{array}{l}3.0 \\
\pm 0.0\end{array}$ & $\begin{array}{l}2.0 \\
\pm 0.0\end{array}$ & $95 \pm 0.1$ & $\begin{array}{l}55 \\
\pm 0.1\end{array}$ & $\begin{array}{l}150 \\
\pm 0.2\end{array}$ & $\begin{array}{l}446 \\
\pm 0.1\end{array}$ & $\begin{array}{l}299 \\
\pm 0.1\end{array}$ & $75 \pm 0.0$ & $\begin{array}{l}8.02 \\
\pm 0.2\end{array}$ & $\begin{array}{l}220 \\
\pm 0.2\end{array}$ \\
\hline 12 & $\begin{array}{l}350 \\
\pm 01 \\
\end{array}$ & $\begin{array}{l}4.7 \\
\pm 0.1\end{array}$ & $\begin{array}{l}2.0 \\
\pm 0.0\end{array}$ & $\begin{array}{l}3.0 \\
\pm 0.0\end{array}$ & $\begin{array}{l}2.0 \\
\pm 0.0\end{array}$ & $95 \pm 0.1$ & $\begin{array}{l}55 \\
\pm 0.1\end{array}$ & $\begin{array}{l}150 \\
\pm 0.2 \\
\end{array}$ & $\begin{array}{l}452 \\
\pm 0.1\end{array}$ & $\begin{array}{l}303 \\
\pm 0.1\end{array}$ & $75 \pm 0.0$ & $\begin{array}{l}8.02 \\
\pm 0.2 \\
\end{array}$ & $\begin{array}{l}220 \\
\pm 0.2 \\
\end{array}$ \\
\hline
\end{tabular}

Natural pond recorded the lowest mean water hardness of $80 \mathrm{mg} / \mathrm{l}$ and the highest mean of 165 $\mathrm{mg} / \mathrm{l}$ was found in collapsible pond. Lowest mean $\mathrm{pH}$ of 6.35 was obtained in natural pond and highest $\mathrm{pH}$ of 8.03 was recorded in collapsible pond. Temperature fluctuations ranged between
29.1 to $35.9{ }^{\circ} \mathrm{C}$ in the ponds. Lowest and highest alkalinity, calcium and magnesium hardness, total water hardness, nitrate, phosphate, electrical conductivity, total dissolved solids, $\mathrm{pH}$ and temperature were obtained in weeks 1 and 12 respectively in all the ponds. 
Mean weekly variations in the water quality of earthen pond

Table 3

\begin{tabular}{|c|c|c|c|c|c|c|c|c|c|c|c|c|c|}
\hline Week & Temp & $\begin{array}{l}\mathrm{DO} \\
\mathrm{mg} / \mathrm{l}\end{array}$ & $\begin{array}{l}\mathrm{CO}_{2} \\
\mathrm{mg} / \mathrm{l}\end{array}$ & $\begin{array}{l}\mathrm{N} \\
\mathrm{mg} / \mathrm{l}\end{array}$ & $\begin{array}{l}\mathrm{P} \\
\mathrm{mg} / \mathrm{l}\end{array}$ & $\begin{array}{l}\mathrm{Ca} \\
\mathrm{mg} / \mathrm{l}\end{array}$ & $\begin{array}{l}\mathrm{Mg} \\
\mathrm{mg} / \mathrm{l}\end{array}$ & $\begin{array}{l}\mathrm{TH} \\
\mathrm{mg} / \mathrm{l}\end{array}$ & $\begin{array}{l}\text { EC } \\
\mu S / c m\end{array}$ & $\begin{array}{l}\text { TDS } \\
\mathrm{mg} / \mathrm{l}\end{array}$ & $\begin{array}{l}\text { Transp } \\
\text { cm }\end{array}$ & pH & $\begin{array}{l}\text { Alk } \\
\mathrm{mg} / \mathrm{l}\end{array}$ \\
\hline 1 & $\begin{array}{l}29.4 \\
\pm 01\end{array}$ & $\begin{array}{l}6.6 \\
\pm 0.1\end{array}$ & $\begin{array}{l}2.0 \\
\pm 0.0\end{array}$ & $\begin{array}{l}1.8 \\
\pm 0.0\end{array}$ & $\begin{array}{l}1.0 \\
\pm 0.0\end{array}$ & $80 \pm 0.1$ & $\begin{array}{l}40 \\
\pm 0.1\end{array}$ & $\begin{array}{l}120 \\
\pm 0.2\end{array}$ & $\begin{array}{l}348 \\
\pm 0.1\end{array}$ & $\begin{array}{l}233 \\
\pm 0.1\end{array}$ & $65 \pm 0.0$ & $\begin{array}{l}6.75 \\
\pm 0.2\end{array}$ & $\begin{array}{l}130 \\
\pm 0.2\end{array}$ \\
\hline 2 & $\begin{array}{l}30.1 \\
\pm 01\end{array}$ & $\begin{array}{l}6.4 \\
\pm 0.1\end{array}$ & $\begin{array}{l}2.0 \\
\pm 0.0\end{array}$ & $\begin{array}{l}1.8 \\
\pm 0.0\end{array}$ & $\begin{array}{l}1.0 \\
\pm 0.0\end{array}$ & $80 \pm 0.1$ & $\begin{array}{l}40 \\
\pm 0.1\end{array}$ & $\begin{array}{l}120 \\
\pm 0.2\end{array}$ & $\begin{array}{l}352 \\
\pm 0.1\end{array}$ & $\begin{array}{l}236 \\
\pm 0.1\end{array}$ & $65 \pm 0.0$ & $\begin{array}{l}6.82 \\
\pm 0.2\end{array}$ & $\begin{array}{l}130 \\
\pm 0.2\end{array}$ \\
\hline 3 & $\begin{array}{l}30.5 \\
\pm 01 \\
\end{array}$ & $\begin{array}{l}6.3 \\
\pm 0.1 \\
\end{array}$ & $\begin{array}{l}1.9 \\
\pm 0.0\end{array}$ & $\begin{array}{l}1.9 \\
\pm 0.0\end{array}$ & $\begin{array}{l}1.2 \\
\pm 0.0\end{array}$ & $85 \pm 0.1$ & $\begin{array}{l}40 \\
\pm 0.1\end{array}$ & $\begin{array}{l}125 \\
\pm 0.2\end{array}$ & $\begin{array}{l}356 \\
\pm 0.1\end{array}$ & $\begin{array}{l}239 \\
\pm 0.1\end{array}$ & $62 \pm 0.0$ & $\begin{array}{l}-6.85 \\
\pm 0.2\end{array}$ & $\begin{array}{l}140 \\
\pm 0.2\end{array}$ \\
\hline 4 & $\begin{array}{l}31.1 \\
\pm 01\end{array}$ & $\begin{array}{l}6.0 \\
\pm 0.1\end{array}$ & $\begin{array}{l}1.9 \\
\pm 0.0\end{array}$ & $\begin{array}{l}1.9 \\
\pm 0.0\end{array}$ & $\begin{array}{l}1.2 \\
\pm 0.0\end{array}$ & $\begin{array}{l}85 \\
\pm 0.1\end{array}$ & $\begin{array}{l}40 \\
\pm 0.1\end{array}$ & $\begin{array}{l}125 \\
\pm 0.2\end{array}$ & $\begin{array}{l}365 \\
\pm 0.1\end{array}$ & $\begin{array}{l}245 \\
\pm 0.1\end{array}$ & $63 \pm 0.0$ & $\begin{array}{l}6.90 \\
\pm 0.2\end{array}$ & $\begin{array}{l}150 \\
\pm 0.2\end{array}$ \\
\hline 5 & $\begin{array}{l}31.7 \\
\pm 01\end{array}$ & $\begin{array}{l}5.9 \\
\pm 0.1\end{array}$ & $\begin{array}{l}1.9 \\
\pm 0.0\end{array}$ & $\begin{array}{l}2.0 \\
\pm 0.0\end{array}$ & $\begin{array}{l}1.3 \\
\pm 0.0\end{array}$ & $90 \pm 0.1$ & $\begin{array}{l}40 \\
\pm 0.1\end{array}$ & $\begin{array}{l}130 \\
\pm 0.2\end{array}$ & $\begin{array}{l}372 \\
\pm 0.1\end{array}$ & $\begin{array}{l}249 \\
\pm 0.1\end{array}$ & $60 \pm 0.0$ & $\begin{array}{l}6.90 \\
\pm 0.2\end{array}$ & $\begin{array}{l}150 \\
\pm 0.2\end{array}$ \\
\hline 6 & $\begin{array}{l}32.3 \\
\pm 01\end{array}$ & $\begin{array}{l}5.7 \\
\pm 0.1\end{array}$ & $\begin{array}{l}1.8 \\
\pm 0.0\end{array}$ & $\begin{array}{l}2.0 \\
\pm 0.0\end{array}$ & $\begin{array}{l}1.3 \\
\pm 0.0\end{array}$ & $90 \pm 0.1$ & $\begin{array}{l}40 \\
\pm 0.1\end{array}$ & $\begin{array}{l}130 \\
\pm 0.2\end{array}$ & $\begin{array}{l}388 \\
\pm 0.1\end{array}$ & $\begin{array}{l}260 \\
\pm 0.1\end{array}$ & $60 \pm 0.0$ & $\begin{array}{l}7.10 \\
\pm 0.2\end{array}$ & $\begin{array}{l}160 \\
\pm 0.2\end{array}$ \\
\hline 7 & $\begin{array}{l}32.7 \\
\pm 01\end{array}$ & $\begin{array}{l}5.6 \\
\pm 0.1\end{array}$ & $\begin{array}{l}1.8 \\
\pm 0.0\end{array}$ & $\begin{array}{l}2.1 \\
\pm 0.0\end{array}$ & $\begin{array}{l}1.4 \\
\pm 0.0\end{array}$ & $90 \pm 0.1$ & $\begin{array}{l}40 \\
\pm 0.1\end{array}$ & $\begin{array}{l}130 \\
\pm 0.2\end{array}$ & $\begin{array}{l}391 \\
\pm 0.1\end{array}$ & $\begin{array}{l}262 \\
\pm 0.1\end{array}$ & $60 \pm 0.0$ & $\begin{array}{l}7.15 \\
\pm 0.2\end{array}$ & $\begin{array}{l}160 \\
\pm 0.2\end{array}$ \\
\hline 8 & $\begin{array}{l}33.6 \\
\pm 01\end{array}$ & $\begin{array}{l}5.5 \\
\pm 0.1 \\
\end{array}$ & $\begin{array}{l}1.8 \\
\pm 0.0\end{array}$ & $\begin{array}{l}2.1 \\
\pm 0.0\end{array}$ & $\begin{array}{l}1.4 \\
\pm 0.0\end{array}$ & $90 \pm 0.1$ & $\begin{array}{l}40 \\
\pm 0.1\end{array}$ & $\begin{array}{l}130 \\
\pm 0.2\end{array}$ & $\begin{array}{l}405 \\
\pm 0.1\end{array}$ & $\begin{array}{l}271 \\
\pm 0.1\end{array}$ & $56 \pm 0.0$ & $\begin{array}{l}7.60 \\
\pm 0.2\end{array}$ & $\begin{array}{l}200 \\
\pm 0.2\end{array}$ \\
\hline 9 & $\begin{array}{l}33.8 \\
\pm 01\end{array}$ & $\begin{array}{l}5.3 \\
\pm 0.1\end{array}$ & $\begin{array}{l}1.8 \\
\pm 0.0\end{array}$ & $\begin{array}{l}2.2 \\
\pm 0.0\end{array}$ & $\begin{array}{l}1.4 \\
\pm 0.0\end{array}$ & $90 \pm 0.1$ & $\begin{array}{l}50 \\
\pm 0.1\end{array}$ & $\begin{array}{l}140 \\
\pm 0.2\end{array}$ & $\begin{array}{l}410 \\
\pm 0.1\end{array}$ & $\begin{array}{l}275 \\
\pm 0.1\end{array}$ & $54 \pm 0.0$ & $\begin{array}{l}7.60 \\
\pm 0.2\end{array}$ & $\begin{array}{l}200 \\
\pm 0.2\end{array}$ \\
\hline 10 & $\begin{array}{l}34.0 \\
\pm 01\end{array}$ & $\begin{array}{l}5.1 \\
\pm 0.1\end{array}$ & $\begin{array}{l}1.7 \\
\pm 0.0\end{array}$ & $\begin{array}{l}2.4 \\
\pm 0.0\end{array}$ & $\begin{array}{l}1.5 \\
\pm 0.0\end{array}$ & $90 \pm 0.1$ & $\begin{array}{l}50 \\
\pm 0.1\end{array}$ & $\begin{array}{l}140 \\
\pm 0.2\end{array}$ & $\begin{array}{l}420 \\
\pm 0.1\end{array}$ & $\begin{array}{l}281 \\
\pm 0.1\end{array}$ & $52 \pm 0.0$ & $\begin{array}{l}7.85 \\
\pm 0.2\end{array}$ & $\begin{array}{l}205 \\
\pm 0.2\end{array}$ \\
\hline 11 & $\begin{array}{l}34.2 \\
\pm 01 \\
\end{array}$ & $\begin{array}{l}5.0 \\
\pm 0.1 \\
\end{array}$ & $\begin{array}{l}1.7 \\
\pm 0.0 \\
\end{array}$ & $\begin{array}{l}2.4 \\
\pm 0.0\end{array}$ & $\begin{array}{l}1.6 \\
\pm 0.0 \\
\end{array}$ & $95 \pm 0.1$ & $\begin{array}{l}50 \\
\pm 0.1\end{array}$ & $\begin{array}{l}145 \\
\pm 0.2 \\
\end{array}$ & $\begin{array}{l}422 \\
\pm 0.1 \\
\end{array}$ & $\begin{array}{l}282 \\
\pm 0.1 \\
\end{array}$ & $52 \pm 0.0$ & $\begin{array}{l}-7.85 \\
\pm 0.2\end{array}$ & $\begin{array}{l}205 \\
\pm 0.2\end{array}$ \\
\hline 12 & $\begin{array}{l}34.8 \\
\pm 01\end{array}$ & $\begin{array}{l}5.0 \\
\pm 0.1\end{array}$ & $\begin{array}{l}1.6 \\
\pm 0.0\end{array}$ & $\begin{array}{l}2.6 \\
\pm 0.0\end{array}$ & $\begin{array}{l}1.6 \\
\pm 0.0\end{array}$ & $95 \pm 0.1$ & $\begin{array}{l}50 \\
\pm 0.1\end{array}$ & $\begin{array}{l}145 \\
\pm 0.2\end{array}$ & $\begin{array}{l}428 \\
\pm 0.1\end{array}$ & $\begin{array}{l}287 \\
\pm 0.1\end{array}$ & $50 \pm 0.0$ & $\begin{array}{l}7.88 \\
\pm 0.2\end{array}$ & $\begin{array}{l}210 \\
\pm 0.2\end{array}$ \\
\hline
\end{tabular}

Mean weekly variations in the water quality of natural pond

Table 4

\begin{tabular}{|c|c|c|c|c|c|c|c|c|c|c|c|c|c|}
\hline Week & Temp & $\begin{array}{l}\mathrm{DO} \\
\mathrm{mg} / \mathrm{l}\end{array}$ & $\begin{array}{l}\mathrm{CO}_{2} \\
\mathrm{mg} / \mathrm{l}\end{array}$ & $\begin{array}{l}\mathrm{N} \\
\mathrm{mg} / \mathrm{l}\end{array}$ & $\begin{array}{l}\mathrm{P} \\
\mathrm{mg} / \mathrm{l}\end{array}$ & $\begin{array}{l}\mathrm{Ca} \\
\mathrm{mg} / \mathrm{l}\end{array}$ & $\begin{array}{l}\mathrm{Mg} \\
\mathrm{mg} / \mathrm{l}\end{array}$ & $\begin{array}{l}\text { TH } \\
\text { mg/l }\end{array}$ & $\begin{array}{l}\text { EC } \\
\mu S / \mathrm{cm}\end{array}$ & $\begin{array}{l}\text { TDS } \\
\mathrm{mg} / \mathrm{l}\end{array}$ & $\begin{array}{l}\text { Transp } \\
\text { cm }\end{array}$ & $\mathrm{pH}$ & $\begin{array}{l}\text { Alk } \\
\mathrm{mg} / \mathrm{l}\end{array}$ \\
\hline 1 & $\begin{array}{l}29.1 \\
\pm 01\end{array}$ & $\begin{array}{l}6.8 \\
\pm 0.1\end{array}$ & $\begin{array}{l}1.6 \\
\pm 0.0\end{array}$ & $\begin{array}{l}1.6 \\
\pm 0.0\end{array}$ & $\begin{array}{l}0.8 \\
\pm 0.0\end{array}$ & $50 \pm 0.1$ & $\begin{array}{l}30 \\
\pm 0.1\end{array}$ & $\begin{array}{l}80 \\
\pm 0.2\end{array}$ & $\begin{array}{l}346 \\
\pm 0.1\end{array}$ & $\begin{array}{l}232 \\
\pm 0.1\end{array}$ & $\begin{array}{l}60 \\
\pm 0.0\end{array}$ & $\begin{array}{l}6.35 \\
\pm 0.2\end{array}$ & $\begin{array}{l}105 \\
\pm 0.2\end{array}$ \\
\hline 2 & $\begin{array}{c}29.8 \\
\pm 0.1 \\
\end{array}$ & $\begin{array}{l}6.8 \\
\pm 0.1\end{array}$ & $\begin{array}{l}1.6 \\
\pm 0.0\end{array}$ & $\begin{array}{l}1.6 \\
\pm 0.0\end{array}$ & $\begin{array}{l}0.8 \\
\pm 0.0\end{array}$ & $50 \pm 0.1$ & $\begin{array}{l}30 \\
\pm 0.1\end{array}$ & $\begin{array}{l}80 \\
\pm 0.2\end{array}$ & $\begin{array}{l}350 \\
\pm 0.1\end{array}$ & $\begin{array}{l}235 \\
\pm 0.1 \\
\end{array}$ & $\begin{array}{l}58 \\
\pm 0.0 \\
\end{array}$ & $\begin{array}{l}6.45 \\
\pm 0.2 \\
\end{array}$ & $\begin{array}{l}105 \\
\pm 0.2 \\
\end{array}$ \\
\hline 3 & $\begin{array}{l}30.1 \\
\pm 0.1\end{array}$ & $\begin{array}{l}6.4 \\
\pm 0.1\end{array}$ & $\begin{array}{l}1.6 \\
\pm 0.0\end{array}$ & $\begin{array}{l}1.6 \\
\pm 0.0\end{array}$ & $\begin{array}{l}1.0 \\
\pm 0.0\end{array}$ & $50 \pm 0.1$ & $\begin{array}{l}30 \\
\pm 0.1\end{array}$ & $\begin{array}{l}80 \\
\pm 0.2\end{array}$ & $\begin{array}{l}356 \\
\pm 0.1\end{array}$ & $\begin{array}{l}239 \\
\pm 0.1\end{array}$ & $\begin{array}{l}54 \\
\pm 0.0\end{array}$ & $\begin{array}{l}6.50 \\
\pm 0.2\end{array}$ & $\begin{array}{l}120 \\
\pm 0.2\end{array}$ \\
\hline 4 & $\begin{array}{l}30.7 \\
\pm 0.1\end{array}$ & $\begin{array}{l}6.3 \\
\pm 0.1\end{array}$ & $\begin{array}{l}1.5 \\
\pm 0.0\end{array}$ & $\begin{array}{l}1.7 \\
\pm 0.0\end{array}$ & $\begin{array}{l}1.0 \\
\pm 0.0\end{array}$ & $50 \pm 0.1$ & $\begin{array}{l}30 \\
\pm 0.1\end{array}$ & $\begin{array}{l}80 \\
\pm 0.2\end{array}$ & $\begin{array}{l}358 \\
\pm 0.1\end{array}$ & $\begin{array}{l}240 \\
\pm 0.1\end{array}$ & $55 \pm 0.0$ & $\begin{array}{l}6.55 \\
\pm 0.2\end{array}$ & $\begin{array}{l}120 \\
\pm 0.2\end{array}$ \\
\hline 5 & $\begin{array}{l}31.3 \\
\pm 0.1 \\
\end{array}$ & $\begin{array}{l}6.0 \\
\pm 0.1\end{array}$ & $\begin{array}{l}1.5 \\
\pm 0.0\end{array}$ & $\begin{array}{l}1.7 \\
\pm 0.0\end{array}$ & $\begin{array}{l}1.0 \\
\pm 0.0\end{array}$ & $50 \pm 0.1$ & $\begin{array}{l}30 \\
\pm 0.1\end{array}$ & $\begin{array}{l}80 \\
\pm 0.2\end{array}$ & $\begin{array}{l}370 \\
\pm 0.1\end{array}$ & $\begin{array}{l}248 \\
\pm 0.1\end{array}$ & $\begin{array}{l}54 \\
\pm 0.0 \\
\end{array}$ & $\begin{array}{l}6.60 \\
\pm 0.2\end{array}$ & $\begin{array}{l}125 \\
\pm 0.2 \\
\end{array}$ \\
\hline 6 & $\begin{array}{r}31.8 \\
\pm 0.1 \\
\end{array}$ & $\begin{array}{l}6.0 \\
\pm 0.1 \\
\end{array}$ & $\begin{array}{l}1.5 \\
\pm 0.0\end{array}$ & $\begin{array}{l}1.8 \\
\pm 0.0\end{array}$ & $\begin{array}{l}1.0 \\
\pm 0.0\end{array}$ & $55 \pm 0.1$ & $\begin{array}{l}30 \\
\pm 0.1\end{array}$ & $\begin{array}{l}85 \\
\pm 0.2 \\
\end{array}$ & $\begin{array}{l}378 \\
\pm 0.1 \\
\end{array}$ & $\begin{array}{l}259 \\
\pm 0.1 \\
\end{array}$ & $\begin{array}{l}51 \\
\pm 0.0 \\
\end{array}$ & $\begin{array}{r}6.92 \\
\pm 0.2 \\
\end{array}$ & $\begin{array}{l}135 \\
\pm 0.2 \\
\end{array}$ \\
\hline 7 & $\begin{array}{l}32.2 \\
\pm 0.1 \\
\end{array}$ & $\begin{array}{l}5.8 \\
\pm 0.1\end{array}$ & $\begin{array}{l}1.5 \\
\pm 0.0\end{array}$ & $\begin{array}{l}1.8 \\
\pm 0.0\end{array}$ & $\begin{array}{l}1.1 \\
\pm 0.0\end{array}$ & $55 \pm 0.1$ & $\begin{array}{l}30 \\
\pm 0.1\end{array}$ & $\begin{array}{l}85 \\
\pm 0.2\end{array}$ & $\begin{array}{l}386 \\
\pm 0.1\end{array}$ & $\begin{array}{l}259 \\
\pm 0.1\end{array}$ & $\begin{array}{l}47 \\
\pm 0.0\end{array}$ & $\begin{array}{r}7.15 \\
\pm 0.2\end{array}$ & $\begin{array}{l}140 \\
\pm 0.2\end{array}$ \\
\hline 8 & $\begin{array}{l}33.0 \\
\pm 0.1\end{array}$ & $\begin{array}{l}5.6 \\
\pm 0.1\end{array}$ & $\begin{array}{l}1.5 \\
\pm 0.0\end{array}$ & $\begin{array}{l}1.8 \\
\pm 0.0\end{array}$ & $\begin{array}{l}1.1 \\
\pm 0.0\end{array}$ & $55 \pm 0.1$ & $\begin{array}{l}30 \\
\pm 0.1\end{array}$ & $\begin{array}{l}85 \\
\pm 0.2\end{array}$ & $\begin{array}{l}394 \\
\pm 0.1\end{array}$ & $\begin{array}{l}264 \\
\pm 0.1\end{array}$ & $\begin{array}{l}44 \\
\pm 0.0\end{array}$ & $\begin{array}{l}7.15 \\
\pm 0.2\end{array}$ & $\begin{array}{l}140 \\
\pm 0.2\end{array}$ \\
\hline 9 & $\begin{array}{l}33.3 \\
\pm 0.1 \\
\end{array}$ & $\begin{array}{l}5.4 \\
\pm 0.1 \\
\end{array}$ & $\begin{array}{l}1.4 \\
\pm 0.0 \\
\end{array}$ & $\begin{array}{l}1.8 \\
\pm 0.0\end{array}$ & $\begin{array}{l}1.1 \\
\pm 0.0\end{array}$ & $55 \pm 0.1$ & $\begin{array}{l}30 \\
\pm 0.1\end{array}$ & $\begin{array}{l}85 \\
\pm 0.2 \\
\end{array}$ & $\begin{array}{l}400 \\
\pm 0.1 \\
\end{array}$ & $\begin{array}{l}268 \\
\pm 0.1 \\
\end{array}$ & $\begin{array}{l}42 \\
\pm 0.0\end{array}$ & $\begin{array}{l}7.20 \\
\pm 0.2 \\
\end{array}$ & $\begin{array}{l}140 \\
\pm 0.2 \\
\end{array}$ \\
\hline 10 & $\begin{array}{l}33.9 \\
\pm 0.1\end{array}$ & $\begin{array}{l}5.3 \\
\pm 0.1\end{array}$ & $\begin{array}{l}1.4 \\
\pm 0.0\end{array}$ & $\begin{array}{l}1.9 \\
\pm 0.0\end{array}$ & $\begin{array}{l}1.2 \\
\pm 0.0\end{array}$ & $55 \pm 0.1$ & $\begin{array}{l}35 \\
\pm 0.1\end{array}$ & $\begin{array}{l}90 \\
\pm 0.2\end{array}$ & $\begin{array}{l}410 \\
\pm 0.1\end{array}$ & $\begin{array}{l}274 \\
\pm 0.1\end{array}$ & $\begin{array}{l}41 \\
\pm 0.0\end{array}$ & $\begin{array}{l}7.40 \\
\pm 0.2\end{array}$ & $\begin{array}{l}150 \\
\pm 0.2\end{array}$ \\
\hline 11 & $\begin{array}{l}34.1 \\
\pm 0.1\end{array}$ & $\begin{array}{l}5.1 \\
\pm 0.1\end{array}$ & $\begin{array}{l}1.4 \\
\pm 0.0\end{array}$ & $\begin{array}{l}2.0 \\
\pm 0.0\end{array}$ & $\begin{array}{l}1.2 \\
\pm 0.0\end{array}$ & $55 \pm 0.1$ & $\begin{array}{l}35 \\
\pm 0.1\end{array}$ & $\begin{array}{l}90 \\
\pm 0.2\end{array}$ & $\begin{array}{l}418 \\
\pm 0.1\end{array}$ & $\begin{array}{l}280 \\
\pm 0.1\end{array}$ & $\begin{array}{l}38 \\
\pm 0.0\end{array}$ & $\begin{array}{l}7.50 \\
\pm 0.2\end{array}$ & $\begin{array}{l}150 \\
\pm 0.2\end{array}$ \\
\hline 12 & $\begin{array}{l}34.5 \\
\pm 0.1 \\
\end{array}$ & $\begin{array}{l}5.0 \\
\pm 0.1\end{array}$ & $\begin{array}{l}1.4 \\
\pm 0.0\end{array}$ & $\begin{array}{l}2.0 \\
\pm 0.0\end{array}$ & $\begin{array}{l}1.2 \\
\pm 0.0\end{array}$ & $55 \pm 0.1$ & $\begin{array}{l}35 \\
\pm 0.1\end{array}$ & $\begin{array}{l}90 \\
\pm 0.2\end{array}$ & $\begin{array}{l}420 \\
\pm 0.1\end{array}$ & $\begin{array}{l}281 \\
\pm 0.1\end{array}$ & $\begin{array}{l}36 \\
\pm 0.0\end{array}$ & $\begin{array}{r}7.50 \\
\pm 0.2\end{array}$ & $\begin{array}{l}160 \\
\pm 0.2 \\
\end{array}$ \\
\hline
\end{tabular}

The concentrations of these parameters increased with time in all the ponds. Highest and lowest concentrations of dissolved oxygen, carbon dioxide and transparency were obtained in weeks 1 and 12 respectively. The concentrations of these parameters decreased with time in all the ponds. There was a significant difference $(P<0.05)$ in the concentrations of dissolved oxygen, carbon dioxide, nitrate, phosphate, alkalinity, calcium and magnesium hardness, total water hardness, $\mathrm{pH}$, electrical conductivity, total dissolved solids, transparency and temperature among the four 
ponds under the different culture systems being practiced in the ponds.

The significant differences in the concentrations of the water quality parameters among the four ponds showed the effects of the different culture systems on the water quality of the ponds. The highest dissolved oxygen concentration in the natural pond was due to the extensive system of culture where the presence of plankton and macrophytes photosynthesis contributed significantly to the high oxygen content. Also, since the pond is fed by rain, interaction of the rain with oxygen in the air as it falls into the pond made oxygen concentrations to be higher especially during the rainy season, as well as proper dissolution and circulation of the atmospheric oxygen by wind. Following the natural pond in term of high oxygen content was the earthen pond where semi-intensive system of culture was practiced. Supplementation of the natural food with artificial food and subsequent decomposition of the artificial feed caused the relatively low oxygen concentration in the pond as compared to the natural pond. The low contents of dissolved oxygen in both concrete and collapsible ponds as compared with natural and earthen ponds was due to the source of the water (borehole or ground water) used in the pond, the materials (concrete and tarpaulin) used for the construction of the ponds, higher temperature in the ponds and the intensive system of culture where artificial feeds were used. The decomposition of the feeds used oxygen, the materials used for the construction did not permit high dissolution and circulation of atmospheric oxygen, while high temperature has been known to decrease oxygen content in water and ground water has been shown to have low dissolved oxygen [7]. In all the ponds, the DO content was of good quality and in the normal range for fish production [8].

The low concentration of carbon dioxide in both natural and earthen ponds was as a result of the utilization of the compound by plankton and macrophytes for photosynthesis. This coming from the extensive and semi-intensive culture systems practiced in the ponds. In case of the collapsible and concrete ponds, the high carbon dioxide content in the ponds was due to the intensive culture system where there was no plankton or macrophytes to consume the carbon dioxide as well as the decomposition of feeds and the respiration of the fish which released $\mathrm{CO} 2$. The high alkalinity content of collapsible and concrete ponds made the ponds to be tolerant of the carbon dioxide [9]. The high $\mathrm{CO} 2$ of the ponds was accompanied by the low dissolved oxygen concentrations as reported by Wurts \& Durborow
(1992) [10]. In all the ponds, the CO2 content was of good quality and in the normal range for fish production [8].

The $\mathrm{pH}$ of natural and earthen ponds which was in the acidic and neutral range probably occurred from the synergistic effects of respiration, photosynthesis and decomposition between plankton, macrophytes, fish and the feed in the case of earthen pond. The low alkalinity and hardness in the ponds could have also contributed to the slight acidity of the ponds. The high alkalinity and total hardness of collapsible and concrete ponds allowed for good buffering capacity of the ponds. This made the $\mathrm{pH}$ in these ponds to be basic. In all the ponds, the $\mathrm{pH}$ fell within the range recommended for fish production [3].

The intensive system which allowed for frequent change of water as well as lime leaching from the concrete pond, the source (ground) water for the ponds may be responsible for the high alkalinity in collapsible and concrete ponds. The low alkalinity in the natural and earthen ponds might have occurred as a result of the semi-intensive and extensive systems where there was no frequent change of water along with processes of respiration and nitrification by plankton and macrophytes. In all the ponds, the alkalinity fell within the range recommended for fish production $[3,10]$.

Calcium and magnesium ions contributed to the total hardness in the ponds with calcium concentration almost twice the concentration of magnesium. The high calcium and magnesium concentrations and hence the total hardness in collapsible and concrete ponds and were due to the source of water (ground) to the ponds, the absence of aquatic plants and the intensive system being practiced. The semi-intensive and extensive systems of culture practiced in earthen and natural ponds which allowed for the growth of aquatic plants could have been the reason for the low calcium and magnesium concentrations in the ponds as the two ions were utilized periodically by the aquatic vegetation. The total hardness in all the ponds could be described as moderate and is within the recommended range for fish culture [10, 11].

The intensive culture system carried out in collapsible and concrete ponds gave rise to the higher transparency recorded among the four pond types. The higher transparency was due to regular flushing of the water, absence of algae, macrophytes, lack of wind stir to cause resuspension of sediments and settling humic substances. The presence of algae, macrophytes, clay, humic and suspended substances were responsible for the low transparency in both 
earthen and natural ponds, this coming from the semi-intensive and extensive culture done in the ponds. However the range of secchi disk transparency recorded in the ponds were able to support fish production. The range of transparency obtained was in agreement with Okonji et al. (2013) who reported that transparency is usually high in intensive culture ponds and low in extensive culture ponds [12].

The relatively high temperature recorded in collapsible and concrete ponds was due to the materials (concrete and tarpaulin) used for the construction of the ponds which absorbs heat, lack of wind action to aid water mixing and the intensive system of culture practiced where there were no algae or macrophytes to serve as heat cover or absorbent. The temperature range in natural and earthen ponds was consequent of the convectional solar radiation coming into the ponds aided by wind action as a result of the exposure of the ponds as well as evaporation of the heat to the atmosphere. The presence of algae and macrophytes due to extensive and semi-intensive system of culture been practiced in the ponds also provided heat cover in the pond and enabled the temperature to be in the range recorded. In all the ponds, the temperature range recorded was optimum for the growth of fish [13].

The system of culture being practiced in the ponds was responsible for the different nitrate and phosphate concentrations recorded in the four ponds. Higher nitrate and phosphate found in collapsible and concrete ponds (where intensive system was done) probably came from the feed which contained high amount of protein. The breakdown of ammonia excreted by the fish, decomposition of uneaten feed and the alkaline medium of the ponds could be responsible for the high nitrate and phosphate concentrations in the ponds. This scenario has been reported by Mustapha \& Akinshola (2016) [14]. The low level of nitrate and phosphate in earthen and natural ponds (where semi-intensive and extensive systems were done) was attributed to the presence of phytoplanktons and macrophytes in the pond which uptake and assimilate the ions in water. The nitrate and phosphate concentrations in all the ponds fell under the permissible level for aquaculture [15].

The conductivity values of the ponds gave a good estimate of the condition of ponds under the different culture systems. Conductivity is an index of the total ionic content of water; while total dissolved solids (TDS) describes all solids (usually mineral salts) that are dissolved in water. Both TDS and electrical conductivity are usually positively correlated. The high conductivity and total dissolved solids in collapsible and concrete ponds probably came from the ground water which was the source of water for the ponds, absence of phytoplankton and aquatic vegetation to assimilate the mineral salts and the effects of residual feed in water which on decomposition added some mineral salts into the water. All these linked to the intensive system of culture in the two ponds. The presence of algae and macrophytes which utilized the salts and rainwater as source of water might be the reason for the slightly lower concentrations of conductivity and TDS in both earthen and natural ponds. The conductivity and TDS range however in the ponds were ideal for fish culture [16]. The conductivity and TDS range of the ponds also confirmed the moderate hardness of the ponds

\section{Conclusions}

The water quality in the four types of ponds under the different culture systems could be described as generally good with most of the water quality parameters falling under the recommended or permissible level for fish culture. There is no pond or culture system that could be described as absolutely perfect in terms of water quality issues. Each of the pond and culture system practiced in the pond has different water quality challenges which could be addressed by carefully looking at the causes and symptoms of the problems to which adequate pond management could be directed. A typical management guide is regular monitoring and measurement of the water quality parameters of ponds to check for abnormalities in the values.

\section{References}

1. Alabaster, J.S., Lloyd, R. (1982). Water Quality Criteria for fresh water fish. London, England, Butterworth Press., 361pp.

2. Boyd, C.E. (1998). Water Quality for Pond Aquaculture. Research and Development Series No. 43. Alabama, International Center for Aquaculture and Aquatic Environments, Alabama Agricultural Experiment, Station, Auburn University., 37pp.

3. Boyd, C.E., Tucker, C.S. (1998). Pond Aquaculture Water Quality Management. Boston, Massachusetts, Kluwer Academic Publishers., 700p.

4. Zweig R.D., Morton, J.D., \& Stewart, M.M. (1999). Source Water Quality for Aquaculture. Washington, The World Bank., 62pp.

5. Bhatnagar, A., Devi, P. (2013). Water quality guidelines for the management of pond fish culture. International Journal of Environmental Sciences, 3(6), 1980-1997, DOI:10.6088/ijes.2013030600019.

6. SAS Institute Inc. (2008). SAS Statistical Software, V.9.2. SAS Institute, Inc. Cary, North Carolina

7. Summerfelt, R.C. (2000). Water Quality Considerations for Aquaculture. Department of Animal Ecology, lowa State University, Ames, USA. 8pp. 
8. Boyd, C.E. (1990). Water Quality in Ponds for Aquaculture. Birmingham, Alabama, Birmingham Publishing Company., 482pp.

9. Hargreaves, J.A., Brunson, M.W. (1996). Carbon dioxide in fish ponds. Starkville, Mississippi, Southern Regional Aquaculture Center, SRAC Publication 468., $6 \mathrm{pp}$.

10. Wurts, W.A., Durborow, R.M. (1992). Interactions of $\mathrm{pH}$, Carbon Dioxide, Alkalinity and Hardness in Fish Ponds, Fact Sheet No. 464. Starkville, Mississippi, Southern Regional Aquaculture Center. 4pp.

11. Wurts, W.A. 2002. Alkalinity and hardness in production ponds. World Aquaculture 33(1), 16-17.

12. Okonji, V.A., Bayoko, T., \& Alufohai, G. (2013). Effects of culture systems on growth and economic performance of Oreochromis niloticus (Linnaeus, 1758) in concrete tanks. African Journal of Biotechnology 12(27), 4374-4379.

13. Delince, G. (1992). The ecology of the fish pond ecosystem, London, England, Kluwer Academic Publishers., 230pp.

14. Mustapha, M., Akinshola, F. (2016). Ammonia concentrations in different aquaculture holding tanks. West African Journal of Applied Ecology, 24(1), 1-8.

15. Pillay, T.V.R. (1992). Aquaculture and the environment. New York, USA, John Wiley and Sons Inc., 189pp.

16. Stone, N.M., Thomforde, H.K. (2004). Understanding Your Fish Pond Water Analysis Report. Cooperative Extension Program, University of Arkansas at Pine Bluff Aquaculture / Fisheries. 4pp. 